\title{
Incorporation of an Amptek Silicon Drift Detector into a Wavelength Dispersive Spectrometer (WDS) Replacing the Gas Flow Proportional Counter
}

\author{
Richard Wuhrer ${ }^{1}$ and Ken Moran ${ }^{2}$ \\ 1. Western Sydney University, Advanced Materials Characterisation Facility, NSW, Australia. \\ 2. Moran Scientific Pty. Ltd, Bungonia, New South Wales, Australia.
}

The Electron Probe Microanalysis (EPMA) instrument has been around for a long time, which utilises the X-ray Microanalysis techniques of Wavelength Dispersive and Energy Dispersive X-ray Spectrometry (WDS and EDS). The WDS design has not changed much since its original development [1]. WDS exhibits far better spectral resolution than the conventional EDS. The combination of EDS and WDS produces a very powerful analytical technique allowing for excellent Quantitative X-ray Mapping (QXRM) [2, 3]. However, the Electron Microprobe operator using WDS, has to be meticulous in monitoring items such as gas flow, gas purity, gas pressure, noise levels of baseline and window, gas flow proportional counter (GFPC) voltage levels, count rate suppression, anode wire contamination and other parameters [2].

Over the last couple years we have been testing the WD spectrometers using Amptek SDD's in place of the sealed proportional counter (PC) and GFPCs [3], see Figure 1a \&1b. Initial work involved using an Amptek pin SDD and more recently the Amptek Fast 123 system. These system configurations include the detector, preamplifier, digital processor and power supplies all in one unit (Figure 1b). More recently, Amptek have produced a different option that allows the detector/preamplifier to be separated from the electronics unit and connected via a flexible cable (Figure1c-g). This allows us to operate the detector remotely from its electronics box. This design option allows the SDD detector to be incorporated within the WD spectrometer and the electronics placed outside using a nine pin feedthrough (Figure 1e \& g).

The incorporation of a SDD, in the light and mid range element range, allows improvement of the detection limit for these elements [3]. It is also possible to obtain much more reliable results at high count rates with a lot less drift in gain and zero.

Our current research demonstrates the dramatic superiority of SDD over PC (whether gas flow or sealed). With a proportional counter it is not really known what X-rays are being counted by the detector. The introduction of a SDD immediately brings to light problems such as secondary fluorescence and other X-rays. With a PC these extra X-rays are similar on the peak as on the background and so are removed by background subtraction, but the $\mathrm{P} / \mathrm{B}$ ratio can be significantly reduced [3]. Most PCs have an order of magnitude poorer energy resolution than the SDD, which means that if interferences are present there will be a corresponding improvement in background by removing them either by energy discrimination or physically removing their source. The superiority of a WDS with a SDD is easily seen once in operation by removing many artefacts allowing for more accurate analysis [5]. 


\section{References:}

[1] K. Moran and R. Wuhrer, $14^{\text {th }}$ Biennial Australian Microbeam Analysis Symposium AMAS (2017) p. 84.

[2] K. Moran and R. Wuhrer, Mikrochimica Acta 155 (2006), p. 59.

[3] R. Wuhrer and K. Moran, Microsc. Microanal. 21 (Suppl 3) (2015), p. 2191.

[4] K. Moran and R. Wuhrer, Microsc. Microanal. 22 (Suppl 3) (2016), p. 92.

[5] The authors wish to thank Amptek for their help in supplying the detectors, in particular John Pantazis.

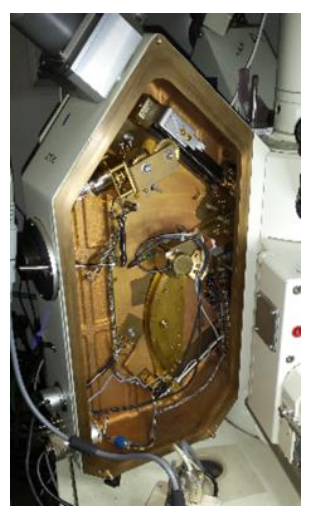

a.

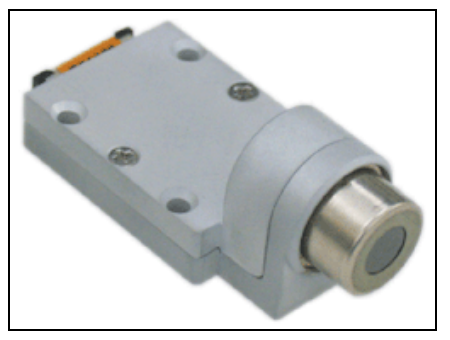

f.

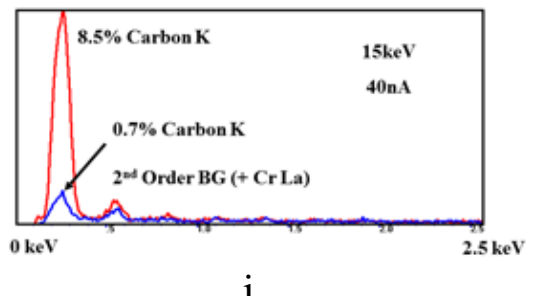

i.

b.
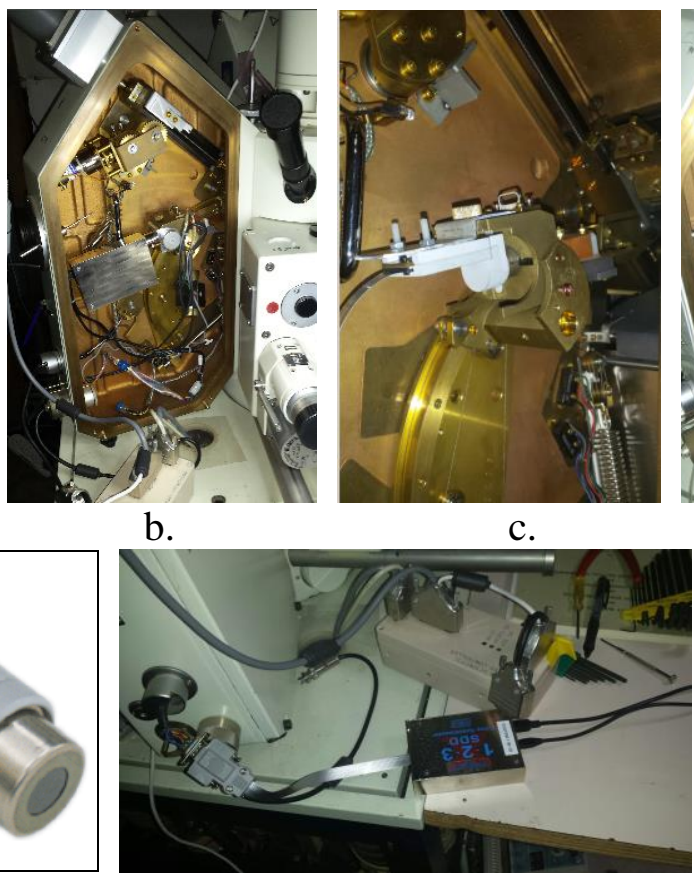

g.

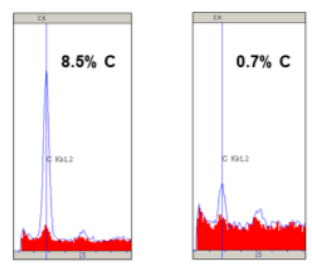

$\mathrm{j}$.

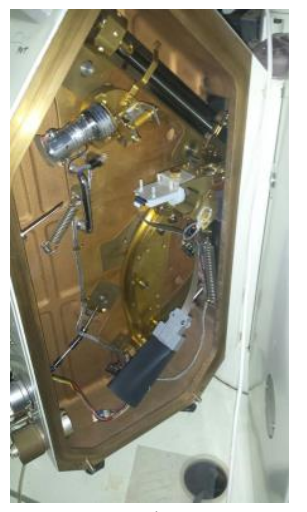

$\mathrm{d}$.

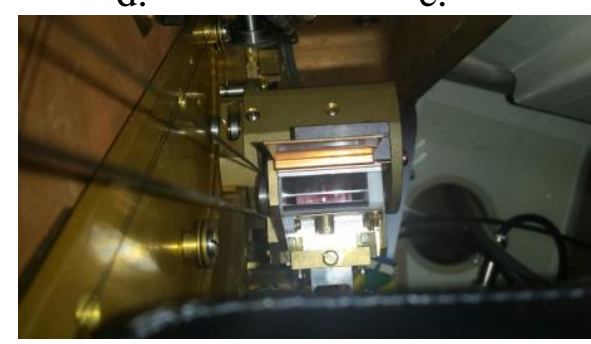

h.

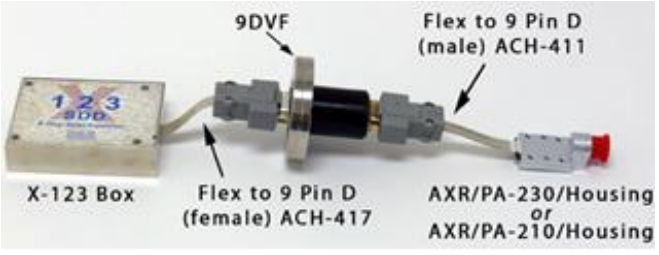

$\mathrm{k}$.

Figure 1. Images of a JEOL WDS with incorporation of a SDD. a) GFPC fitted, b) Amptek mounted in a JEOL spectrometer through an old PC fitting, which has been bored out to allow alignment of the EDS, c) New Amptek configuration SDD, d) electronic wiring, e) feedthrough design, f) Amptek detector, g) external configuration where the detector has been separated from the electronics unit, $h$ ) Amptek SDD viewed through slit design of WDS, i) carbon as measured using an STE crystal at $15 \mathrm{keV}$ 50nA. Blue and Red spectra represent $0.7 \mathrm{wt} \%$ and $8.5 \mathrm{wt} \%[1,4]$. The spectra also include high order diffraction, secondary fluorescence, high-energy electrons and continuum, j) $0.7 \% \mathrm{C}$ and $8.5 \% \mathrm{C}$ spectra from SDD with red being the wavelength background, blue the carbon intensity on peak, and k) New Amptek SDD being incorporated with the control electronics moved to the outside of the WDS. 